Hier steht eine Anzeige. Springer

\title{
Herzrisiko hängt von der Nachbarschaft ab
}

\author{
Forscher der Cleveland Klinik konnten eindrucksvoll belegen, dass \\ eine "unvorteilhafte Nachbarschaft" als Risikofaktor für Herz-Kreislauf- \\ Komplikationen bislang stark unterschätzt wurde.
}

n der Primär- und Sekundärpräven-

tion konzentrierten wir uns in der Vergangenheit häufig viel zu sehr auf die klassischen Risikofaktoren wie Hypertonie, Fettstoffwechselstörung, Nikotinabusus, Diabetes mellitus und Bewegungsarmut. Daneben scheint aber auch der sozioökonomische Status eine Rolle zu spielen.

Die Arbeitsgruppe um Dr. Jarrod Dalton von der Cleveland Clinic in Ohio untersuchte anhand von 110.000 Patienten der Cleveland Klinik in einem mittleren Alter von 56 Jahren den Risikofaktor „sozial benachteiligte Nachbarschaft“. Für ihre Beurteilung verwendeten sie den "Nachbarschafts-DisadvantageIndex“, der Faktoren wie die Anteile der weißen nicht hispanischen Bevölkerung in der unmittelbaren Nachbarschaft enthielt, darüber hinaus den Anteil der Bevölkerung mit Hochschulabschluss, den Anteil der bei Medicaid versicherten Personen und der nicht versicherten Personen, die Anzahl der Haushalte unter der Armutsgrenze und der Haushalte, die von einer "nicht verheirateten Mutter" geführt wurden.

Personen in den Top 5\% (ca. 2.000) waren am stärksten benachteiligt, jene unter den niedrigsten $5 \%$ (20.000) am wenigsten.

Erste waren mit höherer Wahrscheinlichkeit Frauen und hatten häufiger eine dunkle Hautfarbe, einen leicht erhöhten Blutdruck und Diabetes mellitus. Sie hatten auch häufiger eine koronare Herzerkrankung und eine periphere arterielle Verschlusskrankheit.

Die Autoren schlussfolgern, dass das Wohnen in einer sozial benachteiligten Umgebung einen erheblichen Einfluss auf das Herz-Kreislauf-Risiko hat. Wei- tere äußere Faktoren könnten eine Rolle spielen: Luftverschmutzung, Feinstaub, die Möglichkeit, sich gesund zu ernähren, und Zugang zu Fitnessstudios in der Umgebung.

Dalton JE et al. Accuracy of Cardiovascular Risk Prediction Varies by Neighborhood Socioeconomic Position: A Retrospective Cohort Study. Ann Intern Med 2017;167:456-64.

\section{Kommentar}

Neben dem sozioökonomischen Status spielen weitere Faktoren in Zusammenhang mit der Nachbarschaft für das kardiovaskuläre Risiko eine Rolle: Glaubt man der Statistik hier in Deutschland, dann haben fast $40 \%$ aller Deutschen in den letzten Jahren Streit und Auseinandersetzungen mit den Nachbarn. Das macht vielfach Stress und kostet Nerven. Allein $83 \%$ der Deutschen fühlen sich von Straßenlärm belästigt. An zweiter Stelle rangierte dann Fluglärm und Schienenlärm.

Lärm und Stress sind für die Entwicklung von Herz-Kreislauf-Erkrankungen eine fatale Kombination. Das zeigen auch entsprechende Untersuchungen von Kardiologen der Universität Mainz um Prof. Münzel, der den Einfluss von Nachtfluglärm auf die Gefäßfunktion von Patienten mit einer Herzgefäßerkrankung untersuchte. Bei den Teilnehmern stieg der Blutdruck an, die Durchblutung der Gefäße verschlechterte sich und der Schlaf war deutlich gestört.

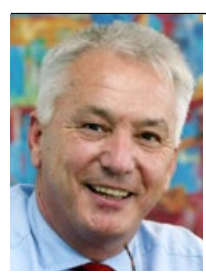

Prof. Dr. med. Curt Diehm Max-Grundig-Klinik, Bühl 